\title{
Directory of Local Chapters
}

During the past year, AMS Council approved the formation of eight new local chapters and reactivated six chapters. The following directory lists all chapters and officers. Chapters are listed by state in alphabetical order. Directories include the year the chapter was founded, year of last election, and a list of the most current officers and addresses, phone and fax numbers, e-mail addresses, and Web addresses (when available). Members who are traveling to any U.S. city where an active local chapter exists should contact that chapter's chairperson for a potential speaking engagement or

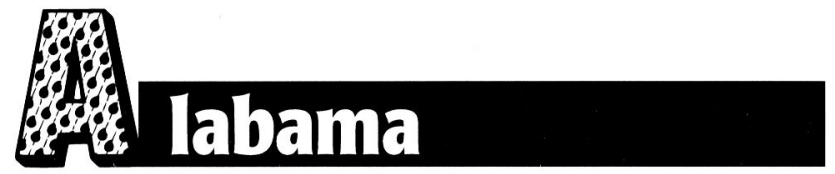

Mobile Bay

\section{Inactive}

\section{University of Alabama In HuntSVILle}

Founded: 1994

Last election: May 1997

President: Jeff Lerner, 1802 Dailey Ter., Huntsville, AL 35816; telephone: 205-922-5970; fax: 205-9225788; e-mail: lerner@atmos.uah.edu

Vice President: Dennis Buechler, 9705 Dertmund Ave., Huntsville, AL 35803; telephone: 205-9225827; fax: 205-922-5788; e-mail: dennis.buechler @msfc.nasa.gov

Secretary: Qu-ling Wu, 650 Wynn Dr., Apt. 279, Huntsville, AL 35816; telephone: 205-922-5779; fax: 205-922-5755; e-mail: wu@vortex.atmos. uah.edu

Treasurer: Kerry Lee Burns, 120 Carnette Dr., Madison, AL 35758-1088; telephone: 205-922-5797; fax: 205-922-5755; e-mail: burns@vortex.atmos. uah.edu

Faculty Advisor: Kevin R. Knupp, 977 Explorer Blvd., Huntsville, AL 35806; telephone: 205-9225762

URL: http://www.atmos.uah.edu/essl/ams/uah _ams_home.html presentation. Members who are interested in forming a new chapter or reactivating a chapter should contact Kelly Garvey, AMS, 45 Beacon St., Boston, MA 02108; telephone: 617-227-2426, ext. 215; e-mail: kgarvey@ametsoc.org or amschap@ametsoc.org. Chapter news should be submitted to Julie Burba, 45 Beacon St., Boston, MA 02108, 617-227-2426, ext. 241; e-mail: jburba@ametsoc.org or chapnews@ametsoc.org. The chapter directory is now located on the AMS Web site (http:// www.ametsoc.org/AMS) and will be updated monthly.

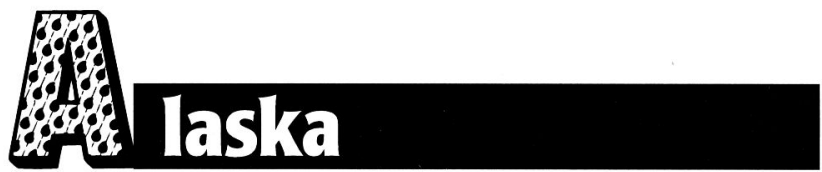

\section{ANCHORAGE}

Founded: 1941

Last election: April 1997

President: Laura Kay Eikermann, 3413 Wisconsin Ave., Anchorage, AK 99517; telephone: 907-2665109; fax: 907-266-5188; e-mail: laura. eikermann@noaa.gov

Vice President: Ann Gravier, 8649 Cross Pointe Loop, Anchorage, AK 99504-2267; telephone: 907-552-2719; fax: 907-552-8351; e-mail: gravier@11afmail.topcover.af.mail

Secretary/Treasurer: Tom Renz, 1414 Helen Dr., Anchorage, AK 99515; telephone: 907-266-5109; fax:907-266-5188; e-mail: trenz@alaska.net or Tom.Renz@noaa.gov

\section{FARTHEST NORTH}

Founded: 1941

Last election: June 1992 (officers reappointed for 1997)

Contact: (Secretary/Treasurer) Ted Fathauer, NWSFO, 101 12th Ave., \#21, Fairbanks, AK 99701 


\section{Central Arizona}

\section{Founded: 1967}

Last election: February 1996

Co-President: David Bjorem, 3405 S. Wilson St., Tempe, AZ 85282; telephone: 602-967-1047; e-mail: A2weather@aol.com

Co-President: Kurt Hill, 521 S. Solomon, Mesa, AZ 85204; telephone: 602-834-5171; e-mail: hill@pvc.maricopa.edu

Secretary: Kathleen O'Connor, 9158 E. Davenport, Scottsdale, AZ 85260; telephone: 602-661-7123; e-mail: oconnor@goodnet.com

Treasurer: Thomas Strache, 15226 N. 61st St., Scottsdale, AZ 85254-2502; telephone: 602-9918980

\section{SOUTHEASt Arizona}

\section{Founded: 1997}

Last election: Spring 1997

President: Darren M. McCollum, 4700 N. Kolb Rd., Apt. \#12108, Tucson, AZ 85750; telephone: 520529-9599; e-mail: darren.mccollum@noaa.gov

Vice President: Douglas P. Boyle, 425 W. Paseo Redondo, \#9B, Tucson, AZ 85701; telephone: 520388-9435; e-mail: boyle@hwr.arizona.edu

Secretary: Kurtis J. Thome, 1481 N. Saddleback Ave., Tucson, AZ 85715; telephone: 520-722-5190; fax: 520-621-4535; e-mail: kurt.thome@opt-sci. arizona.edu

Treasurer: John and Mary Glueck, 13009 N. Meadview Way, Oro Valley, AZ 85737; telephone: 520-544-5632; e-mail: john.glueck@noaa.gov

URL: http:// nimbo.wrh.noaa.gov/Tucson/sear.html

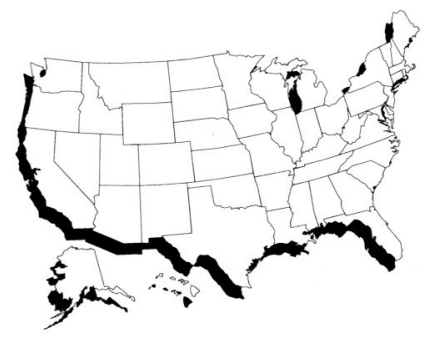

ARKANSAS

Founded: 1995

Last election: January 1997

President: Erwin Prater, 502 W. Bell, Sheridan, AR 72150; telephone: 501-540-3738; fax: 501-5402753; e-mail: prate@infogo.com

Vice President: George R. Wilken, NOAA/NWS, North Little Rock Airport, 8400 Remount Rd., North Little Rock, AR 72118; telephone: 501-8349102, ext.226; e-mail: gwilken@msn.com

Secretary/Treasurer: Chuck Rickard, same address and telephone number as above; $e$-mail: chuckyr@ aol.com

Corresponding Secretary: Newton Skiles, 7800 Toltec Dr., North Little Rock, AR 72116-2838; telephone: 501-835-0790; e-mail: skilesn@ aristotle.net

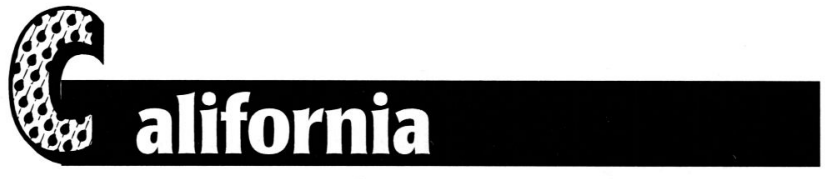

\section{Los ANgeles}

Founded: 1942

Last election: April 1996

Chairman: Jeff Brown, 1100 Rose Ave., Venice, CA 90291; telephone: 310-392-1319; e-mail: 76421.3724@compuserve.com

Vice Chairman: Laura Nunziata, 3205 Los Feliz Blvd., \#11-205, Los Angeles, CA 90039; telephone: 213-665-3535; fax: 213-662-9019; e-mail: 71116.1303@compuserve.com

Secretary: Adrian Ramilliano, 4055 Alla Rd., Los Angeles, CA 90666; telephone: 310-306-7887; e-mail:76450.741@compuserve

Treasurer: Steve LaDochy, Geography Dept., California State Univ., Los Angeles, CA 90032-8222; telephone: 213-343-2222; e-mail: sladoch@ calstatela.edu

\section{Northern California}

\section{Inactive}




\section{RIVERSIDE-SAN BeRnARDino}

Inactive

\section{SACRAMENTO}

\section{Founded: 1955}

Last election: November 1996

Chairman: Donald Baker, 1415 Hickory St., Sacramento, CA 95678; telephone: 916 323-3859; email: dbaker@cleanair.arb.ca.gov

Vice Chairman: MSgt. Rick A. Suggs, 77 OSS/ OSWW, 3028 Peacekeeper Way, Suite 4, McClellan AFB, CA 95652-1020; telephone: 916643-6755; e-mail: SUGGS.RICK @ sma1. mcclellan.af.mil

Secretary/Treasurer: Ronald Lam, NOAA/NWS, 3310 El Camino, Rm. 228, Sacramento, CA 958216308; telephone: 916-979-3041, ext. 511; e-mail: ronald.lam@noaa.gov

\section{SAn Diego}

Founded: 1932

\section{Reactivated: 1996}

\section{Last election: May 1997}

President: Virginia Bigler-Engler, 9739 Caminito Pudregal, San Diego, CA 92123-2105; telephone: 619-694-3298; fax: 619-694-3858; e-mail: wx4engrg@cts.com

Vice President: Armando Garza, 2359 Moonlight Glen, Escondido, CA 92026; telephone: 619-6758700; fax: 619-675-8712; e-mail: armando.garza@ noaa.gov

Secretary: Wilbur Shigehara, 10905 Riesling Dr., San Diego, CA 92131; telephone: 619-271-7258; $e$ mail:wilburshige@worldnet.att.net

Treasurer: Mark Moede, NWS, 11440 W. Bernardo Ct., Suite 230, San Diego, CA 92127-1643; telephone: 619-675-8700; fax: 619-675-8712; e-mail: mark.moede@noaa.gov

\section{San Francisco State University}

\section{Inactive}

\section{San Jose State University}

Founded: 1963

Last election: October 1996

President: Jason Karvelot

Vice President: David Robertson

Secretary: Akriti Shah

Treasurer: Randy Peterson

Sophomore Liaison: Kenneth Craig

ICC Representative: James MacStay

Faculty Advisor: Robert D. Bornstein, Meteorology Dept., San Jose State Univ., 1 Washington Sq., San Jose, CA 95192-0104; telephone: 408-924-5200; fax: 408-924-5191

\section{Santa Barbara/Ventura}

\section{Founded: 1952}

Last election: May 1996

President: Timothy Boyle, Dept. of GeographyWeather Observer, California State Univ., 18111 Nordhoff St., Northridge, CA 91330-8249; telephone: 818-677-5632; fax: 818-677-2723; e-mail: tim.boyle@csun.edu

Vice President: Chris Crabtree, 846 De La Guerra Ter., Santa Barbara, CA 93103; telephone: 805966-0811; fax: 805-963-2431

Secretary: Sally Cappon; telephone: 805-564-5200

Treasurer: Tom Johnston, 5298 Dartworth St., Ventura, CA 93003; telephone: 805-642-7785

\section{University of California, DaVIS}

Founded: 1997

Last election: April 1997

President: Amy Sundquist, 634 Arthur St., Davis, CA 98616-3109; telephone: 916-759-0896; e-mail: afsundquist@ucdavis.edu

Vice President: Colleen Torrenga, 1212 Alvarado Ave., \#33, Davis, CA 95616; telephone: 916-7536467; e-mail: cetorrenga@ucdavis.edu

Secretary: Cynthia Palmer, 1959 Lake Blvd., \#167, Davis, CA 95616; telephone: 916-757-9295; email: ckpalmer@ucdavis.edu

Treasurer: Eric DeFonso, 151 Hoagland Hall, Univ. of California, Davis, Davis, CA 95616; telephone: 916-752-1868; e-mail: defonso@atm24. ucdavis.edu

Faculty Advisor: Su-Tzai Soong, Dept. of Land, Air and Water Resources, Univ. of California, Davis, 
Hoagland Hall, Davis, CA 95616-8627; telephone: 916-752-1406; fax: 916-752-1552

\section{olorado}

\section{Colorado State University}

Founded: 1972

Reactivated: 1996

Last election: 1996

President: Joe Eastman; e-mail: eastman@stromboli. atmos.colostate.edu

VicePresident:Walt Petersen; e-mail: walt@olympic. atmos.colostate.edu

Secretary: Chris Clarke; e-mail: jclarke@taf.atmos. colostate.edu

Treasurer: Larry Carey; e-mail: carey@olympic. atmos.colostate.edu

URL: http://www.atmos.colostate.edu/html/ SocialDir/ams.html

\section{DenVer-BOulder}

\section{Founded: 1938}

\section{Last election: May 1997}

President: Ralph Anderson, 1135 Jay St., Boulder, CO 80302-6914; telephone: 303-541-9288; e-mail: rkaboulder@aol.com

Vice President: Gene J. Pfeffer, 374 Rendezvous Dr., Lafayette, CO 80026; telephone: 303-402-4703; fax: 303-443-1628; e-mail: gene_pfeffer@ radian.com

Secretary: Wendy Abshire, 7465 Holland Ct., Arvada, CO 80005; telephone: 303-423-9722; fax: 303-4978491; e-mail: abshire@ucar.edu

Treasurer: Tim Wilfong, 749 Deer Trail Rd., Boulder, CO 80302; telephone: 303-497-6035; fax: 303497-6978; e-mail: twilfong@etl.noaa.gov

URL: http://www.cgd.ucar.edu/stats/ams/dbams.html

\section{Metropolitan State College of Denver}

Founded: 1991

\section{Reactivated: 1997}

Last election: April 1997

President: Albert Pietrycha, 1040 E. 10th Ave., \#304, Broomfield, CO 80020; telephone: 303-497-8382; fax: 303-497-8401; e-mail: pietrych@ncar.ucar.edu Vice President: Brian Gray, 6009 Yarrow St., Unit E,
Arvada, CO 80004; telephone: 303-420-8021; fax: 303-432-8414; e-mail: sunnygray@idromm.com Secretary: Jennifer Cederle, 516 S. Sherman, \#3, Denver, CO 80209; telephone: 303-733-2695; e-mail: cederle@CGD.ucar.edu

Treasurer: Scott Landolt, 2317 Emery P1., Longmont, CO 80501; telephone: 303-772-4598; fax: 303-4978401; -mail: landolt@ncar.ucar.edu

Faculty Advisor: Anthony Rockwood, Metropolitan State College of Denver, P.O. Box 173362, Denver, CO 80217

\section{PIKes PeAK}

\section{Inactive}

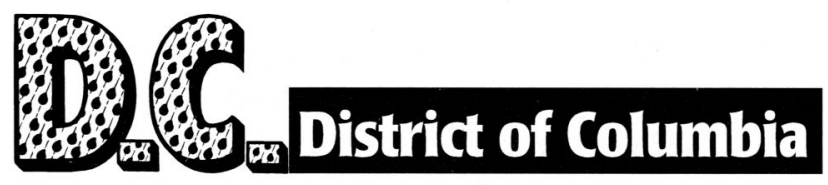

Washington, D.C.

Founded: 1938

Last election: June 1997

Chairperson: Ward R. Seguin, NWS Office of Systems Development, 1325 East-West Hwy., Rm. 12424, Silver Spring, MD 20910; telephone: 301713-1809, ext. 112; e-mail: Ward.Seguin.noaa.gov

Vice Chairperson: Vickie Nadolski, NOAA/NWS, Wx23, Rm. 4348, 1325 East-West Hwy., Silver Spring, MD 20910; telephone: 301-713-2093; e-mail: VickieN@sao.noaa.gov

Corresponding Secretary: Jack Settelmaier, NOAA Science Center, Rm. 706B, 5200 Auth Rd., Camp Springs, MD 20233-9910; telephone: 301-7638151; e-mail:we21sj@hobbes01.wwb.noaa.gov

Recording Secretary: Lauraleen O'Connor, NPOESS/IPO, Centre Bldg, Suite 1450, 8455 Colesville Rd., Silver Spring, MD 20910; telephone: 301-427-2079, ext. 178; e-mail: loconnor@ipo.noaa.gov

Treasurer: Elizabeth Haynes, 4149 25th St., North Arlington, VA 22207-3964; telephone: 703243-4965; e-mail: edhaynes@erols.com

Representative at Large: Sheryl McCarthy, U.S. Dept. of State, Washington, DC 20520; telephone: 202-647-4260; e-mail: smccar2410@aol.com

Chapter Address: D.C. AMS, P.O. Box 13557, Silver Spring, MD 20911-3557

URL: http://wxnet4.nbc4.com/dcams/indexdcams. html 


\section{BIG BEND}

\section{Founded: 1996}

Last election: Pending 1997

Contact: Robert R. Carle, NOAA/NWS, Regional Airport, 3238 Capital Cir., SW, Tallahassee, FL 32310-8723; telephone: 904-576-6318; fax: 904942-9637; e-mail: carle@nws.fsu.edu

\section{Cape Canaveral}

\section{Founded: 1967}

Last election: June 1997

President: Dave Biggar, 45th Weather Squadron, U.S. Air Force, Patrick AFB, FL 32925; telephone: $407-$ 494-7427

Vice President: Steve Hodanish

Secretary: Stephanie Seely

Treasurer: George Diller

URL: http://sunmlb.nws.fit.edu:80/ams.html

\section{FIRST COAST}

\section{Founded: 1996}

Last election: Pending 1997

Contact: Patrick Welsh, Science Operations Officer, NOAA/NWS, 13701 Fang Dr., Jacksonville, FL 32218-7933; telephone: 904-741-4411; fax: 904741-0078; e-mail: pwelsh@unf6.unf.edu

\section{FLoRIDA INSTITUTE OF TECHNOLOGY}

\section{Founded: 1997}

Last election: Pending 1997

Contact: George A. Maul, Florida Institute of Technology, College of Engineering, Division of Marine and Envir. Systems, 150 West Univ. Blvd., Melbourne, FL 32901-6975; telephone: 407-7688000, ext. 8096; fax: 407-984-8461

\section{Florida State University}

Founded: 1948

Last election: April 1997

President: Fiona Horsfall, National Hurricane Center, 11691 S.W. 17th St., Miami, FL 33165; telephone: 305-229-4420; fax: 305-553-5495; e-mail: fiona@nhc.noaa.gov

Vice President: Michelle Huber, same address as above; telephone: 305-229-4421; fax: same as above; e-mail:michelle@nhc.noaa.gov

Treasurer: Terry Fabor, Univ. of Miami, MPO/ RSMAS, 4600 Rickenbacker Cswy., Miami, FL 33149; telephone: 305-361-4885; fax: 305-3614696; e-mail: tfabor@rsmas.miami.edu

\section{Palm Beach County}

\section{Inactive}

\section{West Central Florida}

\section{Inactive}

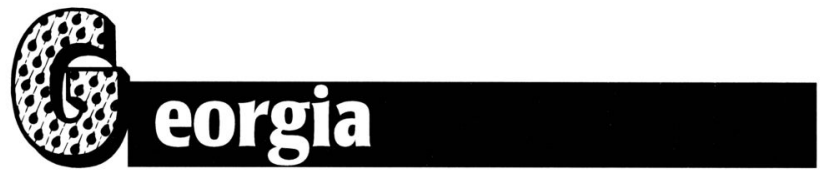

\section{Metropolitan AtLANTA}

\section{Founded: 1951}

Last election: May 1997

President: Jeffrey M. Wilhelm, 1259 Westover Trace, Acworth, GA 30102; telephone: 404-827-2936; fax: 404-827-1560; e-mail: jeff.wilhelm@ turner.com

Vice President: John Wetherbee, 3260 Turtle Lake Crt., Marietta, GA 30067; telephone: 404-9539369; e-mail: BigJohn@Fox97.com

Secretary: I. Jean Graham, 1188 Magnolia Way, Smyrna, GA 30082; telephone: 770-919-5190; e-mail: GaWeather@aol.com

Treasurer: Douglas Fulle, Foster Wheeler Environment Corp., 302 Technology Park, Norcross, GA 30076; telephone: 770-825-7142; fax: 770-8257261; e-mail: atfulled@prodigy.net

\section{Inactive}


Secretary: William J. Johnson, 820 E. Streamwood Blvd., Streamwood, IL 60107-1735; telephone: 630-837-6142

Treasurer: Raymond R. Waldman, 4221 Saratoga, Unit 308A, Downers Grove, IL 60515-1963; telephone: 630-963-3027

Founded: 1961

Reactivated: August 1996

Last election: September 1996

President: Robert W. Farrell, 92-6056 Kalemakapii St., Makakilo, HI 96707; telephone: 808-672-0100; fax: 808-672-0100; e-mail: hiweather@aol.com

Vice President: William H. Little, 94-160 Hokolua Pl., Mililani, HI 96789; telephone: 808-474-6902; fax:808-474-6905; e-mail:u3wxr@cpfemh. cpf.navy.mil

Secretary: Thomas R. Birchard Jr., 1143 Keolu Dr., Kailua, HI 96734; telephone: 808-261-6802; fax: 808-261-6802; e-mail: birchard@ soest.hawaii.edu

Treasurer: Roger V. Pierce, 1289 Lunalilo Home Rd., Honolulu, HI 96825; telephone: 808-973-5280; fax: 808-973-5281; e-mail: Roger.Pierce@noaa.gov

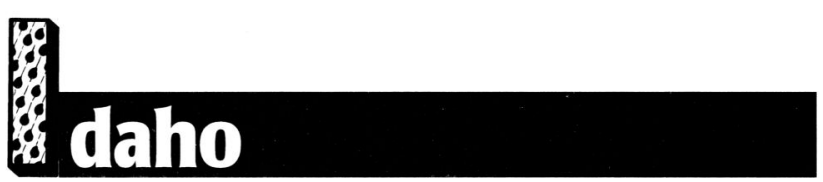

EASTERN IDAHO

URL: http://taiga.geog.niu.edu/nwslot/ams.html

\section{East Central Iluinois}

Founded: 1946

Last election: May 1995

Co-President: Beth Reinke, Illinois State Water Survey, 2204 Griffith Dr., Champaign, IL 61820-7495; telephone: 217-244-2941; fax: 217-244-0220; e-mail: beth@mcc.sws.uiuc.edu

Co-President: Wayne Wendland, same address as above; telephone: 217-333-0729; fax: 217-3336540; e-mail: il@mcc.sws.uiuc.edu

President-Elect: Robert W. Scott, same address as above; telephone: 217-333-4966; fax: 217-3336540; e-mail: r-scott@uiuc.edu

Secretary: Dave Kristovich, same address as above

Treasurer: Mary Schoen Petersen, same address as above; telephone: 217-244-6864; fax: 217-3336540; e-mail:mspetersen@uiuc.edu

\section{NORTHERN ILLINOIS UNIVERSITY}

\section{Founded: 1996}

Last election: Pending 1997

Contact: Robert E. Keislar, U.S. Dept. of Commerce/ NOAA, ARL Field Research Div., 1750 Foote Dr., Idaho Falls, ID 83402-4901

\section{SOUTHWEST IDAHO}

\section{Inactive}

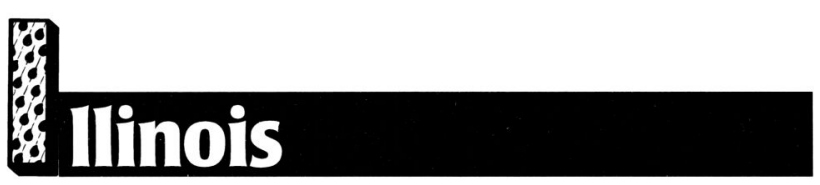

\section{Chicago}

\section{Founded: 1938}

Last election: April 1997

President: Robert J. Hajek, P.O. Box 200, Riverside, IL 60546-0200; telephone: 708-656-0000

Vice President: Alan R. Sealls, 3855 Madison St., Skokie, IL 60076-2803; telephone: 847-329-0225

Founded: 1971

Last election: April 1996

President: Ryan Towell, 1014 E. Chapin, Litchfield, IL 62056; telephone: 217-324-3207; e-mail: towell@geog.niu.edu or rtowell@niu.edu

Vice President: Bill Murrell, 206 Wildflower Pl., Bloomington, IL 61704; telephone: 309-829-2927; e-mail:murrell@geog.niu.edu

Secretary: Kevilee Potts, 4415 Scott Ct., Crystal Lake, IL 60014; e-mail: potts@geog.niu.edu

Treasurer: David Paul, P.O. Box 424, Chadwick, IL 61014; telephone: 815-684-5249; e-mail: paul@geog.niu.edu

Faculty Advisor: David Changnon

Chapter Address: NIU AMS, Northern Illinois Univ., Davis Hall, Rm. 118, DeKalb, IL 60115; telephone: 815-753-0578; e-mail: towell@geog. niu.edu

URL: http://taiga.geog.niu.edu/weather/ams.html 


\section{West Central Iluinois}

Inactive

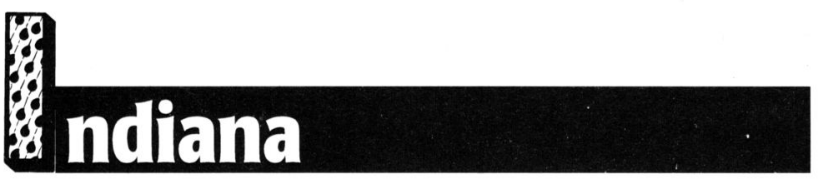

INDIANA

Founded: 1966

Last election: May 1995

President: Lidia E. Dubicki, 6900 W. Hanna Ave., Indianapolis, IN 46241; telephone: 317-856-0360; fax: 317-856-0365; e-mail: 1dubicki@smtpgate. ssmc.noaa.gov

Vice Chairman: Albert P. Shipe, 6900 W. Hanna Ave., Indianapolis, IN 46241; telephone: 317-8560360; fax: 317-856-0365

Secretary/Treasurer: Mark Neyman, 161 West Waterbury Rd., Apt. D, Indianapolis, IN 46217; telephone: 317-232-8252; fax: 317-233-5967

\section{TRI-STATE}

\section{Inactive}

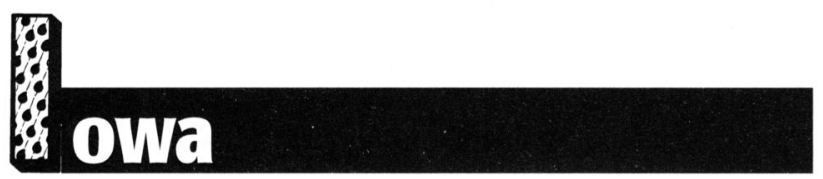

IOWA State University

Inactive

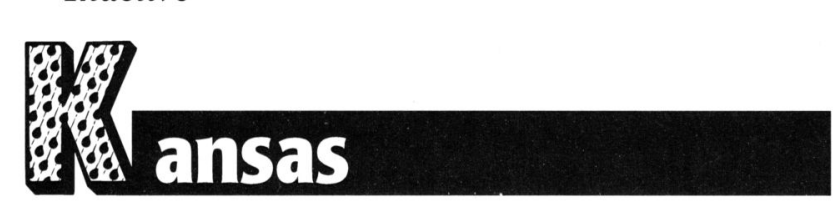

\section{High Plains}

\section{Founded: 1996}

Last election: March 1997

President: Jim J. Johnson, NWS, 104 Airport Rd., Dodge City, KS 67801-9351; telephone: 316-2273311; fax: 316-227-2288; e-mail: jim.johnson@ noaa.gov

Vice President: Matt Gerard, same address and fax number as above; e-mail: matt.gerard@noaa.gov

Secretary: C. Bruce Entwistle, NWS, 920 Armory Rd., Goodland, KS 67735-9273; telephone: 913-
899-7119; fax: 913-899-3501; e-mail: bruce. entwistle@noaa.gov

Treasurer: James Brewster, NWS, 6365 North Osborne Dr. W., Hastings, NE 68901-9120; telephone: 402-462-4287; fax: 402-462-2746; e-mail: james.brewster@noaa.gov

\section{KANSAS UNIVERSITY}

Founded: 1992

Last election: April 1997

President: Lance Steele, 1425 Alumni Pl., Lawrence, KS 66044; telephone: 913-864-6593; e-mail: lsteele@ukans.edu

Vice President: Amy Akers, 2166 W. 26th, \#5, Lawrence, KS 66047; telephone: 913-864-6593; e-mail: akers@falcon.cc.ukans.edu

Secretary/Treasurer: Rod Speer, Rt. 1, Box 256-1, Arkansas City, KS 67005; telephone: 316-4424698; e-mail: rspeer@eagle.cc.ukans.edu

Faculty Advisor: Donna Tucker, Dept. of Physics and Astronomy, Univ. of Kansas, Lawrence, KS 660452151; telephone: 913-864-4738; fax: 913-8645262; e-mail: tucker@phoenix.phsx.ukans.edu

URL: http://chinook.phsx.ukans.edu

\section{South-Central Kansas}

Founded: 1994

Last election: January 1996

President: Richard H. Elder, NWS, 2142 S. Tyler Rd., Wichita, KS 67209; telephone: 316-942-8483, ext. 642

Vice President: Merril D. Teller, KWCH Television, 2815 E. 37th St. N., Wichita, KS 67219; telephone: 316-838-1212; e-mail: weather@kwch.com

Secretary: Mark L. Bogner, KSNW Television, 833 N. Main St., Wichita, KS 67201; telephone: 316265-3333; e-mail: bogner@southwind.net

Treasurer: Dean V. Jones, WeatherData Inc., $245 \mathrm{~N}$. Waco, Suite 310, Wichita, KS 67202; telephone: 316-265-9127 
NORTHWEST LOUISIANA

Inactive

JACKSON PURCHASE

SOUthwest Louisiana And Southeast TeXas

Founded: 1996

Inactive

Last election: Pending 1997

Contact: Chris Jones, 5159 West Brook Blvd., Paducah, KY 42001

\section{KENTUCKY-SOUTHERN INDIANA}

\section{Founded: 1995}

Last election: September 1995

President: Tammy Garrison, WDRB-TV/FOX 41, One Independence Sq., Louisville, KY 40203; telephone: 502-584-6441; fax: 502-589-5559

Vice President: Marvin R. Maddox, 500 Lake Forest Prky., Louisville, KY; telephone: 502-968-4844

Secretary/Treasurer: Mark French, Civil Engineering Dept., Univ. of Louisville, Louisville, KY 40292; telephone: 502-852-4564

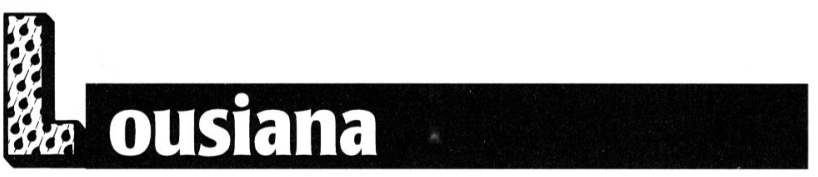

\section{Central Gulf Coast}

\section{Inactive}

\section{Central Louisiana}

\section{Founded: 1986}

\section{Last election: April 1997}

Chairman: Keith Henderson, Dept. of Geography and Anthropology, Louisiana State Univ., Baton Rouge, LA 70803; telephone: 504-388-6137; fax: 504-3882912; e-mail: khenders@maestro.srcc.1su. edu

Vice Chairman: John M. Grymes III, same address as above; telephone: 504-388-6870; fax: same as above; $e$-mail: jgrymes@maestro.srcc.lsu.edu

URL: http://www.srcc.1su.edu/ jgrymes/AMS/

NORTHEAST LoUISIANA UNIVERSITY

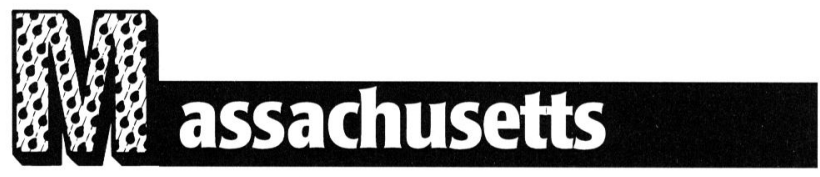

\section{Greater Boston}

Founded: 1929

Last election: February 1996

President: David Towle, 20 Loading Place Rd., Manchester, MA 01944; telephone: 508-526-7109; e-mail: amsdave@prodigy.com

Vice President: Donald A. Towle, 103 Old Essex Rd., Manchester, MA 01944; telephone: 508-526-1062

Treasurer: Rob Gilman, 341 Highland Ave., Quincy, MA 02170; telephone: 617-479-0197 (work), 617471-6980 (home); fax: 617-479-0194; e-mail: 75567.1116@compuserve.com

\section{UnIVERSITY of MasSACHUSETTS-LOWELL}

Founded: 1969

Last election: Spring 1997

President: John Fuller; $e$-mail: fuller@ drizzl.uml.edu

Vice President: Anne Packard; e-mail: packard@ drizzl.uml.edu

Secretary/Treasurer: Dan Fisher; $e$-mail: fisher@ drizzl.uml.edu

Faculty Advisor: Frank P. Colby, Dept. of Earth Sciences, Univ. Massachusetts-Lowell, Lowell, MA 01854; telephone: 508-934-3906; fax: 508-9343003; e-mail: colby@ammetsoc@drizzl.uml.edu

Chapter Address: UMass Lowell Student Chapter of the AMS, UMass Lowell, 35 Standish St., North Campus, Box 154, Lowell, MA 01854; telephone: 508-934-3910; e-mail: ammetsoc@drizzl.uml.edu

\section{Inactive}


SOUTHEAST MichIGAN

\section{Founded: 1965}

Last election: October 1996

President: Martin R. Kaufman, 1515 E. Hurd Rd., Monroe, MI 48162; telephone: 313-289-3541

Vice President: Dick Wagenmaker, 9200 White Lake Rd., White Lake, MI 48386; telephone: 248-6253309, ext. 766; fax: 248-625-4834; e-mail: richard.wagenmaker@noaa.gov

Secretary: Dan Reilly, same address as above; telephone: 248-625-3309; fax: same as above; e-mail: daniel.reilly@noaa.gov

Treasurer: Richard Foltman, 6244 Cambridge St., Dearborn Heights, MI 48127; telephone: 313-2356185; fax: 313-235-8053; e-mail: foltmanr@ detroitedison.com

URL: http://www.crh.noaa.gov/dtx/semcams.htm

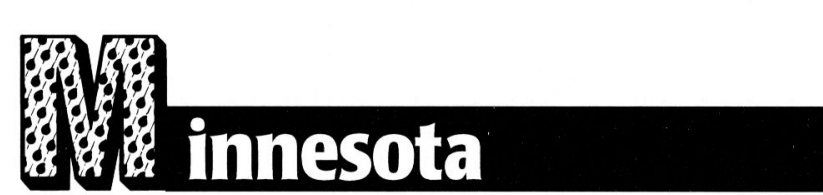

\section{Twin Cities}

\section{Founded: 1948}

Last election: May 1997

President: Craig Edwards, 18625 Jacques Ct., Eden Prairie, MN 55346; e-mail: Craig.Edwards@ noaa.gov

Vice President: Bob Conzemius, KEYC-TV, 1570 Lookout Dr., Mankato, MN 56003; e-mail: bconzemi@ic.mankato.mn.us

Secretary/Treasurer: Chris Orr, Kavouras, Inc., 11400 Rupp Dr., Burnsville, MN 55337; telephone: 612-890-0609; fax: 612-882-4500; e-mail: corr@kavouras.com

URL: http://Byte.stthomas.edu/www/math_http/ weather/tcametsoc.html

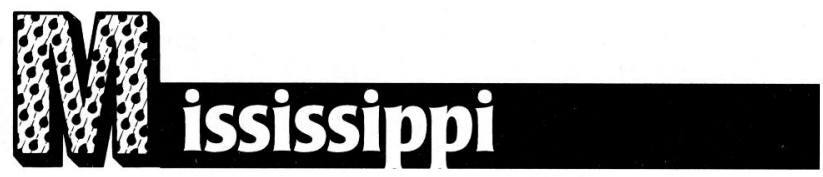

\section{Central Gulf Coast}

Founded: 1989

Last election: September 1996

President: William H. Burnett, 1020 Balch Blvd., Stennis Space Center, MS 39529-5005; telephone: 601-688-4766; fax: 601-688-5332; e-mail: 532@CNMOC.NAVY.MI

Vice President: Lt. James Rasure, same address as above; telephone: 601-688-5750; fax: same as above; e-mail: N321@CNMOC.NAVY.MIL

Secretary/Treasurer: James Price, same address as above; telephone: 601-688-4859; fax: 601-6885791

\section{JACKSON}

Founded: 1990

Reactivated: 1996

Last election: November 1996

President: Paul J. Croft, Dept. of Atmospheric Sciences, Jackson State Univ., P.O. Box 17660, Jackson, MS 39217-0460; telephone: 601-968-7012; e-mail:pcroft@stallion.jsums.edu

Vice President: Alan Gerard, NWS, 234 Weather Service Dr., Jackson, MS 39208; telephone: 601936-2189; e-mail: Alan.Gerard@noaa.gov

Secretary: Rusty Pfost, same adresses and telephone number as above; telephone: 601-936-2189; e-mail: Rusty.Pfost@noaa.gov

Treasurer: Patrick Fitzpatrick, Dept. of Physics and Atmospheric Sciences, Jackson State Univ., P.O. Box 17660, Jackson, MS 39217-0460; telephone: 601-968-7012; e-mail: fitz@ riscman.jsums.edu

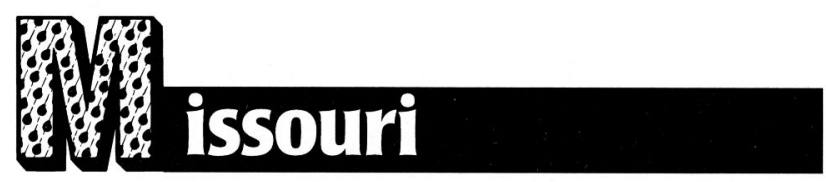

Greater St. Louis

Founded: 1945

Last election: May 1997

Chairman: Wayne E. McCollom, 1606 Bermuda Dr., O'Fallon, IL 62269-2981; telephone: 618-632-8387; e-mail: mccollom@apci.net 
Vice Chairman: Jim Moore, Dept. of Earth and Atmospheric Sciences, Saint Louis Univ., 3507 Laclede Ave., St. Louis, MO 63103; telephone: 314-977-3126; e-mail: moore@eas.slu.edu

Secretary: Susan Tarbell, Air Weather Service Technical Library, 859 Buchanan St., Scott AFB, IL 62225; telephone: 618-256-2625; fax: 618-2563772;e-mail: tarbells@thunder.safb.af.mil

Treasurer: Karan T. Hofmann, 109 Longmeade Dr., O'Fallon, IL 62269-7023; telephone: 618-256-8406; fax: 618-628-0238; e-mail: hofmannk@transcom. safb.af.mil

\section{Kansas CitY}

\section{Founded: 1934}

Last election: April 1997

President: Patricia L. Cooper, 732 SE Pierre Ave., Lee's Summit, MO 64063; telephone: 816-5540971

Vice President: Samuel K. Beckman, 9311 Willow Rd., Liberty, MO 64068; telephone: 816-792-0217

Secretary: James H. Henderson, 13801 Scottie Dr., Kearney, MO 64060; telephone: 816-781-5694

Treasurer: Heide Petermann, 9235 Somerset Dr., Overland Park, KS 66207-2479; telephone: 913381-9336

URL: http://www.crhnwscr.noaa.gov/eax/eax.htm

\section{SAINT LOUIS UnIVERSITY}

Founded: 1974

Last election: April 1996

President: Michael S. Norato, Saint Louis Univ., 3700 W. Pine Blvd., Fusz Hall, Box 105, St. Louis, MO 63108; telephone: 314-977-4542; fax: 314-9774542; e-mail: noratoms@slu.edu

Vice President: Mary Koleda, Saint Louis Meteorology, 3507 Laclede Ave., St. Louis, MO 63103; telephone: 314-291-1765; e-mail: koleda@eas.slu.edu

Secretary: Michael A. Durdock, Griesedieck Hall, 3630 W. Pine Blvd., St. Louis, MO 63108; telephone: 314-977-4323; e-mail: durdockm@slu.edu

Treasurer: Nathaniel J. White, 3700 W. Pine Blvd., St. Louis, MO 63108; telephone: 314-977-4841; fax: 314-977-4841; e-mail: whitenj@ slu.edu
ОМАНA-OFFUTT

Founded: 1955

Last election: April 1997

Chairman: Brian Smith, NWS Forecast Office, 6707 N. 288 St., P.O. Box 719, Valley, NE 68064-0719; telephone: 402-289-3422

Vice Chairman: Bruce Telfeyan, Air Force Global Weather Ctr., Offutt AFB, NE 68113

Corresponding Secretary: Jeff Doran, same address as above; telephone: 402-294-1684; e-mail: doranj@afgw.offutt.af.mil

Recording Secretary: Dave Skerritt, NWS Forecast Office, 6707 N. 288 St., P.O. Box 719, Valley, NE 68064-0719; telephone: 402-289-3422

Treasurer: John Gray, Air Force Global Weather Ctr., Offutt AFB, NE 68113

URL: http://sundog.creighton.edu/ ams/amspage. html

\section{UnIVERSITY OF NEBRASKA AT LINCOLN}

Founded: 1994

Last election: April 1997

President: Russ Bigley

Vice President: Jeremy Duensing

Secretary: Amanda Adams

Treasurer: Doug Behne

Faculty Advisor: Michael Palecki, Univ. of Nebraska, Dept. of Geog., 324 Avery Hall, Lincoln, NE 68588-0135; telephone: 402-472-6657; fax: 402472-1185; e-mail: mpalecki@unlinfo.unl.edu

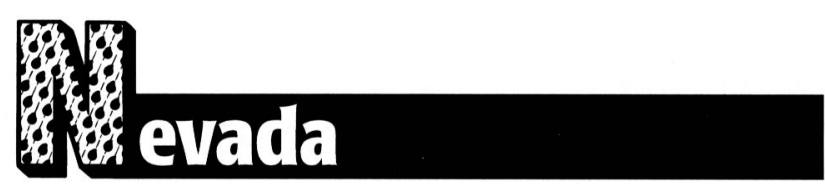

\section{NoRTHERn NEVADA}

Founded: 1969

Last election: June 1996

President: Steve Adams, NWS, 2350 Raggio Pkwy., Reno, NV 89512-3900; telephone: 702-673-8107 (work), 702-322-7602 (home)

Vice President: Sherri Del Soldato, 1601 Calusa Ln., Reno, NV 89523; telephone: 702-746-4649 
Secretary: Roger Lamoni, same address as above; telephone: 702-673-8100

Treasurer: Douglas French, 3755 Wilcox Ranch Rd., Reno, NV 89510

\section{SOUTHERn Nevada}

\section{Inactive}

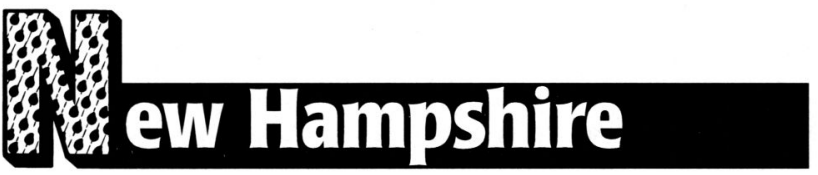

\section{Plymouth State College}

\section{Founded: 1988}

\section{Last election: April 1997}

President: Eric Chaves, PSC Suite \#1623, 19 Highland Ave., Plymouth, NH 03264; telephone: 603535-7150; $e$-mail: e_chaves@oz.plymouth.edu

Vice President: Christopher McAloon, PSC Suite \#3221, 19 Highland Ave., Plymouth, NH 03264; telephone: 603-535-7147; e-mail: c_mcaloo@oz. plymouth.edu

Secretary: James Rufo, PSC Suite \#3942, 19 Highland Ave., Plymouth, NH 03264; e-mail: j_rufo@oz.plymouth.edu

Treasurer: Jason Shafer, PSC Suite \#4630, 19 Highland Ave., Plymouth, NH 03264; telephone: 603535-7160; e-mail: j_shafer@oz.plymouth.edu

Faculty Advisor: Ashton Peyrefitte Jr., Natural Science Dept., Boyd Hall, Rm. 111, Plymouth State College, Plymouth, NH 03264-1519; telephone: 603-535-2321

Chapter Address: AMS, PSC Suite \#A21, 19 Highland Ave., Plymouth, NH 03264

URL: http://oz.plymouth.edu:80/ meteo/home.html

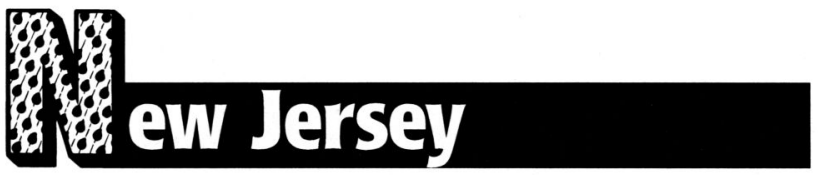

\section{Cook College Rutgers University}

Inactive

\section{Kean College}

Founded: 1960

Last election: April 1995

Co-President: Carolyn Mupo
Co-President: Tom Else

Secretary: Gil Rozett

Treasurer: Chester Schmitt

Chapter Address: Dept. of Meteorology, Kean College of New Jersey, Union, NJ 07083

\section{New Jersey}

\section{Inactive}

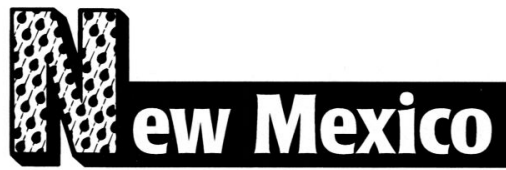

\section{El Paso-Las Cruces}

\section{Founded: 1957}

Last election: February 1997

President: Gail-Tirrel Vaucher, AMSRL-IS-EA, Army Research Lab., White Sands Missile Range, NM 88002-5501; telephone: 505-678-3237; fax: 505-678-3385; e-mail: gvaucher@arl.mil

Vice President: David Knapp, same adress as above; telephone: 505-678-8148; fax: 505-678-3385; e-mail:dknapp@arl.mil

Secretary/Treasurer: James Reynolds, 1279 Desierto Seco Dr., El Paso, TX 79912-1136; telephone: 915581-9028; fax: 505-589-4026; e-mail: James. Reynolds@NOAA.GOV

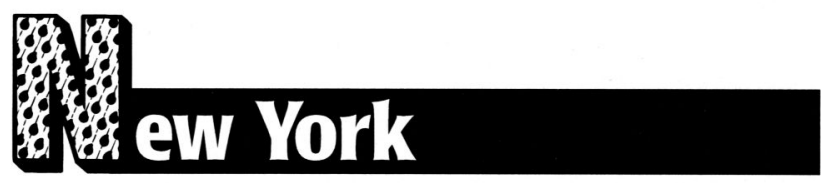

\section{Central New York}

Founded: 1986

Last election: June 1997

Co-President: David Eichorn, WIXT-TV 9, 5904 Bridge St., East Syracuse, NY 13057; telephone: 315-446-4780

Co-President: Mark W. Wysocki, Dept. of Soil, Crop and Atmospheric Sciences, Cornell Univ., Bradfield Hall, Rm. 1114, Ithaca, NY 14853; telephone: 607-255-3034; e-mail: WYSOCKI@metvax.cit. cornell.edu

Vice President: John Ferlito, 73 Lawrence St., Oswego, NY 13126; telephone: 315-343-5801

Secretary: Kathy Orr, WTVH 5, 9080 James St., Syracuse, NY 13203; telephone: 315-425-5555

Treasurer: Al Stamm, Dept. of Earth Sciences, 
SUNY at Oswego, Piez Hall, Oswego, NY

13126; telephone: 315-341-2799

\section{City College of New York}

\section{Inactive}

\section{Cornell University}

Founded: 1979

Last election: April 1997

Co-President: Deena Bollinger, Cornell Univ., Bradfield Hall, Rm. 1114, Ithaca, NY 14853; telephone: 607-255-3034; fax: 607-255-2106

Co-President: Michelle Tuorto, same address and telephone and fax numbers as above

Secretary: Maura Drnevich, same address and telephone and fax numbers as above

Treasurer: Matt Noyes, same address and telephone and fax numbers as above

Faculty Advisor: Mark Wysocki, Cornell Univ., Bradfield Hall, Rm. 1114, Ithaca, NY 14853; telephone: 607-255-2568; fax: 607-255-2106; e-mail: wysocki@metvax.cit.cornell.edu

\section{INTERIOR OF EASTERN NeW YoRK}

\section{Inactive}

\section{New York City/Long IsLand}

\section{Founded: 1932}

Last election: May 1997

Chairperson: Mark L. Kramer, c/o Meteorological Evaluation Services Co., 165 Broadway, Amityville, NY 11701; telephone: 516-691-3395; fax: 516-691-3550; e-mail: mesamity@li.net

Vice President: Frank P. Castelli, same address and telephone and fax numbers as above; e-mail: mesamity@li.net

Secretary: Jeffrey S. Tongue, 175 Brookhaven Ave., Upton, NY 11973; telephone: 516-924-0593, ext. 224; fax: 516-924-0519; e-mail: jeffrey.tongue@ noaa.gov

Treasurer: Samual Abrahamer, 3271 Benjamin Rd., Oceanside, NY 11572; telephone: 516-766-7843

URL: http://www.nws.bnl.gov/meetings.html
State University of New York

\section{AT BROCKPORT}

Founded: 1978

Last election: Spring 1997

President: Jerod C. McIntyre; e-mail: jmcintyr@ weather.brockport.edu

Vice President: Debby Slocum; e-mail: dslocum@ weather.brockport.edu

Secretary: Thomas Mahoney; e-mail: tmahoney@ weather.brockport.edu

Treasurer: Jeff Andrews; e-mail: jandrews@ weather.brockport.edu

Chapter Address: SUNY at Brockport Student Chapter of the AMS, 102 Lennon Hall, Brockport, NY 14420-2936; telephone: 716-395-2636; fax: 716$395-2416$

URL: http://www.weather.brockport.edu:80/ams/

\section{State University of New York}

AT ONEONTA

Founded: 1982

Last election: April 1997

President: David Hart

Vice President: Todd Gutner

Secretary/Treasurer: Joshua Maloy

Faculty Advisor: Jerome Blechman, Meteor. Club, Dept. of Earth Sciences, SUNY at Oneonta, Oneonta, NY 13820-4015; telephone: 607-4363707; fax: 607-436-3547

\section{State University of New York at Oswego}

\section{Inactive}

\section{Western New York}

Founded: 1949

\section{Last election: May 1997}

President/Treasurer: Andy Ross, 76 Wardman Rd., Kenmore, NY 14217; telephone: 716-876-3354

Vice President/Secretary: Warren Glover, 17 Newport Ave., Buffalo, NY 14216; telephone: 716-8362903 
箘 orth Carolina

ASHEVILLE

\section{Inactive}

\section{Central North Carolina}

Founded: 1968

Last election: April 1997

President: Jerry H. Crescenti, 211 Trillingham Ln., Cary, NC 27513; telephone: 919-481-4539 (home), 919-541-4567(work); e-mail: crescenti.jerry @epamail.epa.gov

Vice President: Chris D. Thompson, 1113 Medlin Dr., Cary, NC 27511; telephone: 919-467-5154 (home), 919-821-8720 (work); e-mail: wxguy@ interpath.com

Secretary: Mark D. Huncik, 600 Occoneechee Dr., Fuquay-Varina, NC 27526; telephone: 919-5578134 (home), 919-362-3258 (work); e-mail: mark.huncik@cplc.com

Treasurer: Paula J. Kurilla, 4509 South Ridge Dr., Fuquay-Varina, NC 27526; telephone: 919-5576668 (home), 919-515-8211 (work); e-mail: paula.kurilla@noaa.gov

\section{Greater Charlotte}

\section{Inactive}

North Carolina State University

Founded: 1969

Last election: April 1997

President: Nate Johnson

Vice President: John Goff

Secretary: Jennifer Hehl

Treasurer: Jill Derby

Faculty Advisor: Jerry Watson, AMS Student Chapter, MEAS, North Carolina State Univ., Box 8208, Raleigh, NC 27695; telephone: 919-515-7076; fax: 919-515-7802

\section{University of North Carolina \\ at AsheVILLE}

Founded: 1986

Last election: April 1996

President: Henry Reges, 135 Furman Ave., Apt. \#6, Asheville, NC 28801; e-mail: reges@atms. unca.edu

Vice President: Stuart Hinson; e-mail: hinson@ atms.unca.edu

Secretary: Steve Taylor; e-mail: staylor@vortex. atms.unca.edu

Treasurer: Tim Armstrong

Faculty Advisor: Edward Brotak, Atmospheric Sciences Dept., Univ. of North Carolina at Asheville, Attn: ATMSCI, 1 University Hts., Asheville, NC 28804; telephone: 704-255-6442; e-mail: ebrotak@uca.edu

URL: http://vortex.atms.unca.edu/ams/index.html

\section{\% orth Dakota}

LAKE AGASSIZ

\section{Inactive}

\section{LAKOTA CHAPTER}

Founded: 1996

Last election: February 1997

President: Viggo Jensen, NWS/Forecast Office, P.O. Box 1016, Bismarck, ND 58502-1016; telephone: 701-250-4495; fax: 702-250-4450

Vice President: John P. Martin, same address as above; telephone: 701-250-4224; fax: 702-2504450; e-mail: John.Martin@ noaa.gov

Secretary: Tanja Fransen, same address as above; telephone: 701-250-4224; fax: 702-250-4450; e-mail: tanja.fransen@noaa.gov

Treasurer: Darin Langerud, North Dakota Atmospheric Resource Board, 900 E. Boulevard Ave., Bismarck, ND 58505-0851; telephone: 701-3282788; e-mail: dlanger@water.swc.state.nd.us 


\section{NORTH DAKOTA}

\section{Founded: 1980}

Last election: April 1996

President: Shane Wood, P.O. Box 9006, Grand Forks, ND 58202; telephone: 701-772-4205; e-mail: shwood@badlands.nodak.edu

Vice President: Charles Bell, 408 17th St. NW, \#23, East Grand Forks, MN 56721; telephone: 218-7736780; e-mail: chbell@plains.nodak.edu

Secretary: Renae Onstad, 203B Brannon Hall, Grand Forks, ND 58202; e-mail: ronstad@badlands. nodak.edu

Treasurer: Raymond Romanski; e-mail: romanski@badlands.nodak.edu

Faculty Advisor: Leon Osborne, Dept. of Atmospheric Sciences, P.O. Box 9006, Grand Forks, ND 58202

URL: http://www.aero.und.edu:80/Student-Organizations/AMS/index.html

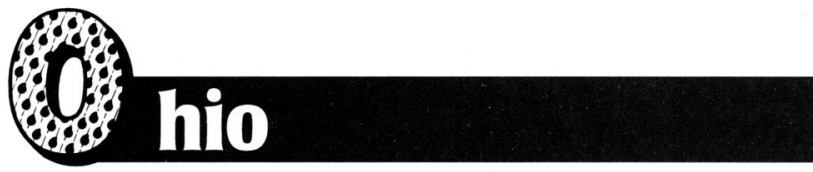

\section{Cincinnati}

\section{Founded: 1991}

Last election: April 1996

President: Daniel P. Mullen, 7980 Green View Ct., West Chester, OH 45069; telephone: 513-777-4036

Vice President: Rich Apuzzo, WXIX-TV, 635 W. 7th St., Cincinnati, OH 45203; telephone: 513-4210119; fax: 513-421-3022

Secretary: Julie Dian, NWS, 1901 S. St., Rt. 134, Wilmington, $\mathrm{OH} 45177$

Treasurer: Jack Nebergall

\section{NORTHEAST OHIO}

\section{Founded: 1984}

Last election: February 1997

President: Matt Higgins, 13900 Quail Oval, North Royalton, $\mathrm{OH} 44133$; telephone: 216-237-8498

Vice President: Eric Wertz, 3529 Dayton Ave., Kent, $\mathrm{OH}$ 44240; telephone: 216-673-3783

Secretary: Paul DiGiorgio, 2978 Ridgeline Trail, Stow, OH 44224; telephone: 330-678-4805; e-mail: PaliDg@Newreach.com
Treasurer: Dick Vadar, 22250 Riverwalk Rd., Rocky River, OH 44116; telephone: 216-331-7465

URL: http://www.csuohio.edu/NWS

\section{NORTHWest OHIO}

Founded: December 1993

Last election: December 1995

President: Robert Dale, 3816 Hoiles, Toledo, $\mathrm{OH}$ 43612; telephone: 419-476-9275; fax: 419-8415561; e-mail: rdale@norden1.com

Vice President: N/A

Secretary/Treasurer: Chad Ruppel

URL: http://norden1.com/ rdale/ams.html

\section{Wright Memorial}

\section{Founded: 1960}

Last election: October 1996

President: Jason Tuell, AFIT, 703 Sykes Cir., WPAFB, OH 45433-1331

Vice President: Tom Neu, AFMC/DOW, WPAFB, $\mathrm{OH} 45433$; telephone: 513-257-5365; e-mail: neut@wpgate1.wpafb.af.mil

Secretary/Treasurer: Pat Hayes, 88th Weather Squadron, 2049 Monahan Way, Bldg. 91, WPAFB, OH 45433-7204; telephone: 513-255-2207; e-mail: hayes@tamu.edu

URL: http://weather.lab.wpafb.af.mil/ams/

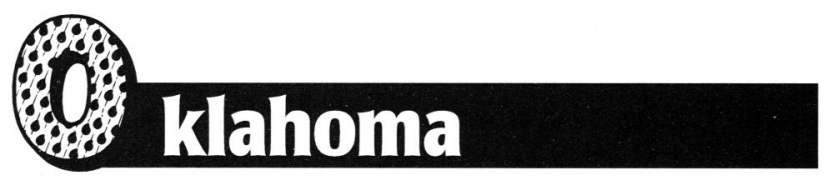

\section{Central Oklahoma}

Founded: 1959

Last election: May 1997

President: Kit K. Wagner, Atmospheric Information Systems, P.O. Box, 721165, Norman, OK 73070; telephone: 405-329-8707; fax: 405-329-8717; e-mail: kit@ionet.net

Vice President: Kelvin Droegemeier, Center for Analysis and Prediction of Storms, Univ. of Oklahoma, 100 E. Boyd St., Suite 1110, Norman, OK 73019; telephone: 405-325-0453; fax: 405-3257614; e-mail: kdroege@ou.edu

Secretary: Harold J. Keeney, WSR-88D Operational Support Facility, 1200 Westheimer Dr., Norman, OK 73069; telephone: 405-366-6560, ext. 4272; 
fax: 405-366-6557; e-mail: Harold.J.Kenney@ noaa.gov

Treasurer: Charlie A. Crisp, National Severe Storms Laboratory, 1313 Halley Cir., Norman, OK 73069; telephone: 405-366-0448; fax: 405-366-0472; e-mail: charlie.crisp@noaa.gov

URL: http://www.nssl.noaa.gov/cocams

\section{NoRTHEAST OKLAHOMA}

Founded: 1993

Last election: Spring 1997

President: James Aydelott, KO-TV, P.O. Box 6, Tulsa, OK 74104

Vice President: David Bernard, KJRH-TV, 3701 S. Peoria, Tulsa, OK 74135; telephone: 918-7481580; fax: 918-748-1436; e-mail: bernard@kjrh.com

Secretary/Treasurer: Bryan Osborne, NWS, Arkansas-Red River Basin, Forecast Center, 10159 E. 11th St., Suite 300, Tulsa, OK 74128-3050; telephone: 918-832-4109; fax: 918-832-4101; e-mail: Bryan.Osborne@noaa.gov

\section{UNIVERSITY OF OKLAHOMA}

Founded: 1956

Last election: April 1997

President: Christopher Duvall, Oklahoma Univ. Chapter, c/o School of Meteorology Energy Center, Rm. 1310, 100 E. Boyd St., Norman, OK 73019-0470; telephone: 405-325-6561; e-mail: gandalf@ou.edu

Vice President: Amy Talmage, same address and telephone number as above; $e$-mail: atalmage@ rossby. metr.ou.edu

Secretary: Gavin Essenberg, same address and telephone number as above; e-mail: gessenberg@ ou.edu

Treasurer: Laura Lemanski, same address and telephone number as above; $e$-mail: lemansky@ rossby. metr.ou.edu

URL: http://www.ou.edu/student/tornado/

\section{OREGON}

Founded: 1947

Last election: January 1996

President: Jim Little, KOIN-TV, 222 SW Columbia, Portland, OR 97201; telephone: 503-464-0706; fax: 503-464-0806

Vice President: Chuck Weise

Secretary: Mark Nelsen, 36006 Historic Columbia River Hwy., Corbett, OR 97019; telephone: 503695-5823

Treasurer: Ron Thorkildson, 6338 SW 175th Pl., Aloha, OR 97007; telephone: 503-230-4168; fax: 503-230-3984

Councilors: George Stephan, Ivy Mclane, George Miller, and Dan Keaton

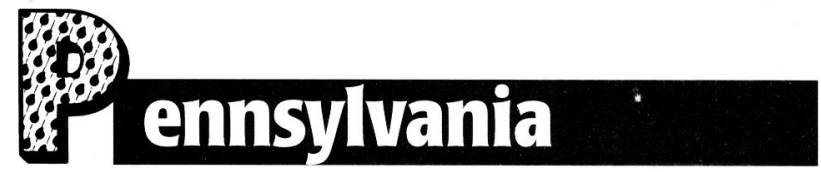

Central Pennsylvania

Inactive

Greater Pittsburgh

Inactive

Millersville University

Founded: 1986

Last election: April 1997

President: Jeff Weinrich, 233B Diehm Hall, Millerville Univ., P.O. Box 1002, Millersville, PA 17551-0302; e-mail:jweinric@ rain.millersv.edu

Vice President: Sam Ng; e-mail: sng@rain. millergy.$e d u$

Secretary: Joe Bartosik; e-mail: jbartosi@rain. millersv.edu

Treasurer: Bonnie Rollins, 508B Burrowes Hall, Millerville Univ., P.O. Box 1002, Millersville, PA 17551-0302 ; e-mail: brollins@rain.millersv.edu 


\section{Pennsylvania State University}

Founded: 1952

Last election: April 1997

President: James West, c/o Hans Verlinde, 503 Walker Bldg., University Park, PA 16802; telephone: 814-865-0478; fax: 814-865-3663; e-mail: west@psumeteo.psu.edu

Vice President: Steve Strum, same address as above; e-mail: strum@psumeteo.psu.edu

Secretary/Treasurer: Andrew DeCandis, same address as above; $e$-mail: ajd150@psu.edu

Faculty Advisor: Hans Verlinde, 503 Walker Bldg., University Park, PA 16802

\section{outh Carolina}

\section{Palmetto}

Founded: Reactivated 1988

Last election: April 1997

President: Sandi St. Claire, 1201 Main St., Suite 1100, Columbia, SC 29201; telephone: 803-737-0800; fax: 803-765-9080; e-mail: stclaire@water.dnr. state.sc.us

Vice President: Jim Gandy, WIS Television, P.O. Box 367, Columbia, SC 29202; telephone: 803-7991010; fax: 803-758-1278; e-mail: JimGandy@ aol.com

Secretary/Treasurer: Robert Buckley, Westinghouse Savannah River Company, Savannah River Technology Ctr., Bldg. 773A, A1008, Aiken, SC 29808; telephone: 803-725-1926; fax: 803-725-4233; e-mail: robert.buckley@srs.gov

\section{South Carolina Low Country}

\section{Inactive}

\section{Upstate South Carolina}

Founded: 1994

Last election: September 1996

Chariman: Thomas Johnstone, NWS, 1549 GSP Dr., Cincinnati, N., International Airport, Greer, SC 29615; e-mail: thomas.johnstone@noaa.gov

Vice Chairman: Carlton Ulbrich, Dept. of Physics and Astronomy, Clemson Univ., Clemson, SC
29634-1911; telephone: 803-656-5322; fax: 803656-0805; e-mail: cwu@clouds.phys.clemson.edu

Secretary: Richard Neal, 114 Plantation Dr., Greer, SC 29651; telephone: 803-848-9971; fax: 803-8485072

Treasurer: Jeff Zoltowski, NWS, 1549 GSP Dr., Cincinnati, N., International Airport, Greer, SC 29615 URL: http://tgsv5.nws.noaa.gov/er/gsp/ams.htm

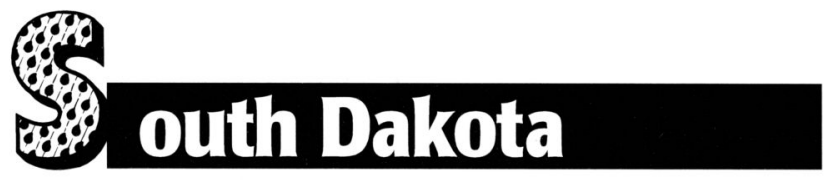

\section{SiouxLand Weather MINDs}

Founded: September 1994

Last election: November 1996

President: Larry M. Browning, 1102 Second St., Brookings, SD 57006; telephone: 605-692-4089; fax: 605-688-5878; e-mail: browninl@mg. sdstate.edu

Vice President: Julia C. Vetter, 809 Hermosa Dr., Sioux Falls, SD 57104; telephone: 605-330-4247; fax: 605-330-4248; e-mail: jcvetter@aol.com

Secretary: Tim Masters, 3100 S. Glendale, Sioux Falls, SD 57105; telephone: 605-331-6404; fax: 605-330-4248; e-mail: timothy.masters@noaa.gov

Treasurer: Arthur D. Umland, 46274 Park Pl., Hartford, SD 57033-6720; telephone: 605-526-6463; fax: 605-330-4248; e-mail: artsd@aol.com

Chapter Address: Weather MINDs, P.O. Box 90002 , Sioux Falls, SD 57109-1011

URL: http://physics.sdstate.edu/wx_minds/wxminds. htm

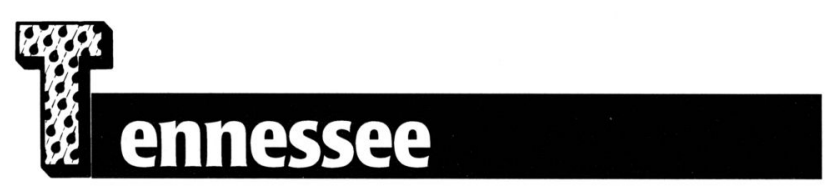

\section{MEMPHIS}

Founded: 1970

Last election: May 1996

Chairperson: Mark Isaminger, Lincoln Laboratory, MIT, 3385 Airways Blvd., Suite 233, Memphis, TN 38116-3808; telephone: 901-346-0214; fax: 901346-0517; e-mail: marki@1l.mit.edu

Vice Chairperson: Richard Smith, NWS Memphis WSFO, 7777 Walnut Grove Rd., OM-1, Memphis, TN 38120-2198; telephone: 901-757-6441; fax: 901-757-6435 
Secretary: Ben Boorman, Lincoln Laboratory, MIT, 3385 Airways Blvd., Suite 233, Memphis, TN 38116-3808; telephone: 901-346-0214; fax: 901346-0517; e-mail: benb@11.mit.edu

Treasurer: Tom Amis, CWSU-ARTCC, 3229 Democrat Rd., Memphis, TN 38116; telephone: 901-3688528; fax: 901-368-8105

URL: http://www.srh.noaa.gov/meg/ams.html

\section{Tennessee Valley}

Founded: 1988

Reactivated: 1997

Last election: May 1997

Chairperson: Barry Clay Roberts, P.O. Box 269, St. Joseph, TN 38481; telephone: 205-244-6124; e-mail: barry.roberts@msfc.nasa.gov

Vice Chairperson: Eugene W. McCaul, 977 Explorer Blvd., Huntsville, AL 35806; telephone: 205-9225837; fax: 205-922-5788; e-mail: bill.mccaul@ msfc.nasa.gov

Secretary: Anthony R. Guillory, same address as above; telephone: 205-922-5894; fax: 205-9225723; e-mail: anthony.guillory@msfc.nasa.gov

Treasurer: Dan Schmit, 4611 Governors House Dr., 207, Huntsville, AL 35805; telephone: 205-5336397; e-mail:dschmit@traveller.com

URL: http://www.atmos.uah.edu/ess1/ams/uah _ams_home.html

\section{\%ge? \\ exas}

Alamo

Founded: 1981

Last election: January 1997

President: Larry Peabody, 11611 Caprock, San Antonio, TX 78230-2102; telephone: 210-558-3906; fax: 210-696-7246; $e$-mail: trenk@connecti.com

Vice President: John Willing, KMOL-TV, P.O. Box 2641, San Antonio, TX 78299; telephone: 210-2264444; fax: 210-224-9898

Secretary: Nezette Rydell, 4130 San Antonio, TX 78217; telephone: $210-656-6562$

Treasurer: Robert A. Blaha, 119 Riviera, San Antonio, TX 78213; telephone: 210-341-1191

\section{Central Texas}

Founded: 1948

Last election: May 1994 (officers reappointed for 1997)

President: Bob Rose, c/o Lower Colorado River Authority, P.O. Box 220, Austin, TX 78767; telephone: 512-473-3299; fax: 512-473-3299

Vice President: N/A

Secretary/Treasurer: Arney Srackangast

Chapter Address: Central Texas Chapter of the AMS, c/o Bob Rose, Lower Colorado River Authority, P.O. Box 220, Austin, TX 78767

\section{Houston}

\section{Founded: 1965}

Last election: May 1997

President: Cecilia Sinclair, KRIV-TV, 3935 Westheimer, Houston, TX 77027; telephone: 713625-1973; e-mail: rsanvido@interserv.com

Vice President: Robert Van Hoven, NWS, 1620 Gill Rd., Dickinson, TX 77539; telephone: 281-3375074; e-mail: robert.vanhoven@noaa.gov

Secretary: Aaron Studwell, Source Environmental, Inc., 4100 Westheimer, Suite \#106, Houston, TX 77027; telephone: 713-621-4474; e-mail: jenaaron@starbase.neosoft.com

Treasurer: Doris Rotzoll, Spaceflight Meteorology Group, Johnson Space Center, Houston, TX 77058; telephone: 281-483-1041; e-mail: drotzoll@ gp801.jsc.nasa.gov

URL: http://www.srh.noaa.gov:80/ftproot/hgx/html/ index $1 / \mathrm{ams} 1 / \mathrm{ams} 1 . \mathrm{htm}$

\section{NORTH TEXAS}

Founded: 1937

Last election: May 1996

President: Jerri Johnson, 309 W. 15th St., Irving, TX 75060

Vice President: Bernard N. Meisner, NWS Southern Region Headquarters, 819 Taylor St., Rm. 10A26, Ft. Worth, TX 76102; telephone: 817-334-2671; fax: 817-334-3475; e-mail: bmeisner@smtpgate. ssmc.noaa.gov

Secretary: Gary R. Woodall, same address as above; telephone: 817-334-2812; fax: 817-334-4920; e-mail: gwoodall@smtpgate.ssmc.noaa.gov

Treasurer: Steven G. Cooper, same address as above; 
telephone: 817-334-2652; fax: 817-334-4920;

e-mail: scooper@smtpgate.ssmc.noaa.gov

\section{Permian Basin}

Founded: 1994

Last election: Pending 1997

Contact: Raymond R. Fagen, MIC, NWS, 2500 Challenger Dr., Midland, TX 79706; telephone: 915563-5006; fax: 915-563-8117

\section{TEXAs A\&M UnIVERSITY}

\section{Founded: 1956}

Last election: April 1997

President: Lori Grimm, 603 Southwest Pkwy., \#101, College Station, TX 77840; telephone: 409-6968121; e-mail:1grimm@nimbus.tamu.edu

Vice President: Jason Jordan; e-mail: stormchaser@ tamu.edu

Secretary: Julie Jasien, 410 S. Texas Ave., College Station, TX 77840; e-mail: jaj9728@unix.tamu.edu

Treasurer: Sam Shamburger; e-mail: samsham@ tamu.edu

Faculty Advisor: Mike Biggarstaff, TAMU, Dept. of Meteorology, College Station, TX 77843-3150

URL: http://tamu.edu/ jkj1852/TAMSCAMS/

\section{WeST TEXAs}

Founded: February 1993

Last election: April 1996

President: Scott J. Carpenter, 227 Indiana Ave., Apt. C210, Lubbock, TX 79415; telephone: 806-7400627; e-mail:m9sjc@ttacs.ttu.edu

Vice President: John L. Schroeder

Secretary: Kyle A. Hill, 2619 19th St., Apt. \#4, Lubbock, TX 79410; e-mail: m9kah@ttacs.ttu.edu

Treasurer: David R. Deighan, 5502 49th St., Apt. \#14, Lubbock, TX 79414; telephone: 806-7914634; e-mail:wadrd@ttacs.ttu.edu

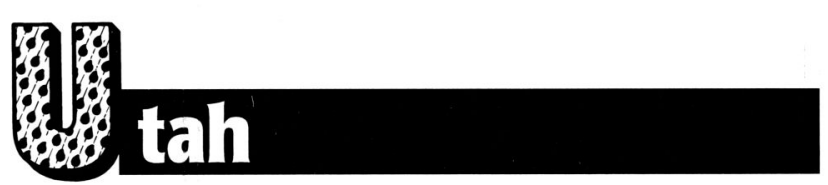

UTAH

Founded: 1942

Last election: May 1997

President: James Nelson, NWSFO, 2242 W. North
Temple, Salt Lake City, UT 84116; telephone: 801524-5133; fax: 801-524-4030; e-mail: James. Nelson@NOAA.GOV

Vice President: Randy Graham, same address and telephone and fax numbers as above; e-mail: Randall.Graham@NOAA.GOV

Treasurer: Peter Wilensky, same address and telephone and fax numbers as above; $e$-mail: Peter.Wilensky@NOAA.GOV

Publicity Chair: Larry Nierenber, same address and telephone and fax numbers as above; e-mail: Larry.Nierenberg@NOAA.GOV

URL: http://nimbo.wrh.noaa.gov/AMS/

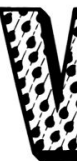

\section{ermont}

\section{Lyndon State College}

Founded: 1971

Last election: April 1996

President: Gerry Bielinski, Box 7522, Lyndon State College, Lyndonville, VT 05851; telephone: 802626-6661; fax: 802-626-9770 (ATTN: LSC-AMS); e-mail: bielinskig@queen.lsc.usc.edu

Vice President: Sean Hickey, Box 7654, Lyndon State College, Lyndonville, VT 05851; telephone: 802262-6541; fax: 802-626-9770 (ATTN: LSC-AMS); e-mail: hickeys@queen.lsc.usc.edu

Secretary: Brian Whitley, Box 7283, Lyndon State College, Lyndonville, VT 05851; telephone: 802626-6665; fax: 802-626-9770 (ATTN: LSC-AMS); e-mail: whitleyb@queen.lsc.usc.edu

Treasurer: Jim Longmuir, Box 7713, Lyndon State College, Lyndonville, VT 05851; telephone: 802626-6641; fax: 802-626-9770 (ATTN: LSC-AMS); e-mail: longmuirj@queen.lsc.usc.edu

Faculty Advisor: Bruce Berryman, Meteor. Dept., Lyndon State College, Lyndonville, VT 05851

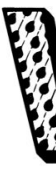

\section{Central Virginia}

Founded: 1983

Last election: Spring 1996

President: Todd Anderson, Virginia Power, 5000 Dominion Blvd., Glen Allen, VA 23060; telephone: 
804-273-3008; fax: 804-273-3410; e-mail: Todd_Anderson@vapower.com

Vice President: Dan Salkovitz, Virginia Dept. of Environmental Quality, P.O. Box 10009, Richmond, VA 23240-0009; telephone: 804-698-4404; fax: 804-698-4510; e-mail: ddsalkovit@deq.state. va.us

Treasurer: Todd Anderson, Virginia Power, 5000 Dominion Blvd., Glen Allen, VA 23060; telephone: 804-273-3008; fax: 804-273-3410; e-mail: Todd_Anderson@vapower.com

\section{HAMPTON Roads}

\section{Inactive}

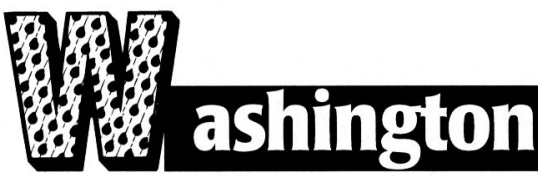

\section{Puget SOUND}

\section{Founded: 1935}

Last election: April 1996

President: Randy Cryer, 2925 Fairview Ave. E., Apt. 8, Seattle, WA 98102-3055

Vice President: Don Atkinson, Dept. of Atmospheric Science, Univ. of Washington, Box 351640, Seattle, WA 98195-1640; telephone: 206-543-6290; $e$ mail: ona@atmos.washington.edu

Secretary/Treasurer: Jordan Sutton, P.O. Box 3463, Everett, WA 98203-8463; telephone: 206-3380943

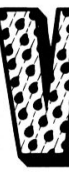

\section{Greater Milwaukee}

Founded: 1973

Last election: January 1997

President: Michal K. Thomas, 922 Park St., Apt. 107, Oregon, WI 53575-2890; telephone: 608-8356407; e-mail: mkthoma1@ facstaff.wisc.edu

Vice President: James M. Frederick, 2108 Butler Dr., Waukesha, WI 53186; telephone: 414-547-6954; e-mail: jmf@alpha1.csd. uwm.edu

Secretary: Michael Westendorf, $7110 \mathrm{~W}$. Chambers Ct., \#2, Milwaukee, WI 53210; telephone: 414-4491469; e-mail: Westrice@csd.uwm.edu
Treasurer: Maribeth Scheel, 409 Nautica Dr., Port Washington, WI 53074; telephone: 414-284-4748

\section{Northeast Wisconsin}

\section{Inactive}

\section{UNIVERSITY OF WISCONSIN-MADISON}

Founded: 1987

Last election: May 1997

President: Steve Hale, 1225 W. Dayton St., Madison, WI 53706

Vice President: Tim Czerwonka, 1225 W. Dayton St., Madison, WI 53706

Secretary: Kim Schneider, 1225 W. Dayton St., Madison, WI 53706

Treasurer: Jason Vandervest, 1225 W. Dayton St., Madison, WI 53706;

Faculty Advisor: David D. Houghton, Rm. 1525, 1225 W. Dayton St., Madison, WI 53706

URL: http://www.meteor.wisc.edu/uw-aos/amsuwmad.html

\section{WeSTERn Wisconsin}

Founded: October 1991

Last election: Fall 1996

President: John Racy, 403 Gillette St., Apt. \#204, LaCrosse, WI 54603; telephone: 219-447-6317

Vice President: Dan Baumgardt, W453 Sunshine Dr., Stoddard, WI 54658; telephone: 608-784-8275

Secretary/Treasurer: Cory Malles, WKBT-TV, 141 S. 6th St., LaCrosse, WI 54601; telephone: 608784-7897

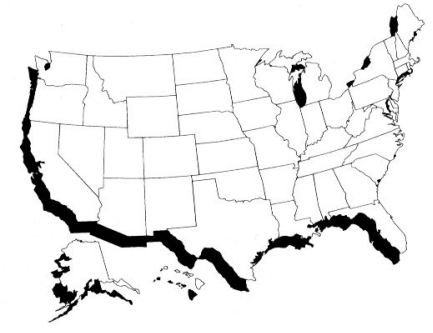


SOUTEAST WYOMING

Inactive

\section{uerto Rico}

\author{
Puerto Rico
}

Founded: February 1996

Last election: Pending 1997

Contact: Ada R. Monzon, P.O. Box 9066594, San Juan, PR 00906-6594

\section{New Chapters}

Big Bend-Florida

Eastern Idaho-Idaho

First Coast-Florida

Florida Institute of Technology-Florida

Jackson Purchase-Kentucky

Southeast Arizona-Arizona

University of California, Davis - California

Puerto Rico-Puerto Rico

\section{Reactivated Chapters}

\author{
Aloha-Hawaii \\ Colorado State University - Colorado \\ El Paso-Las Cruces-New Mexico \\ Jackson-Mississippi \\ Metropolitan State College of Denver-Colorado \\ Tennessee Valley-Tennessee
}

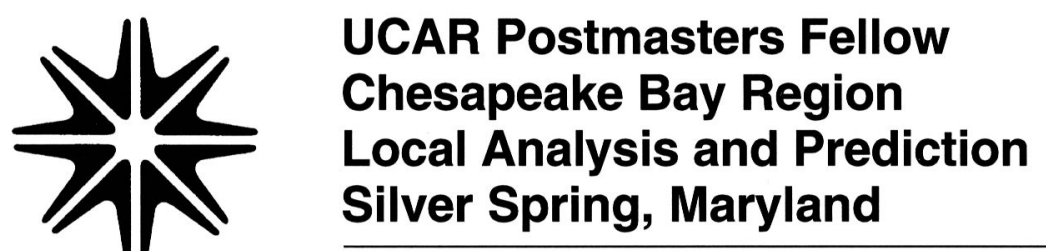

UCAR is seeking a Postmasters Fellow to be located at the Coast Survey Development Laboratory of the National Ocean Service working with investigators to develop, implement, and maintain the Local Analysis and Prediction System (LAPS) for the Chesapeake Bay area.

This project is part of a larger endeavor to force an oceanographic forecast model of the Chesapeake Bay with high-resolution atmospheric analyses and forecasts. The selected candidate will be responsible for obtaining meteorological data to be used in LAPS and for the set up and maintenance of the LAPS. This task will involve interfacing with government agencies and private sources of data, obtaining permission and operational access to those data, and documenting the results. In addition, the candidate will assist in implementation of the LAPS for the Chesapeake Bay region, be responsible for its maintenance and for operational dissemination of its products to a wide community of users, and maintain a Chesapeake LAPS website.

Candidates should have a masters degree (or higher) in ocean or atmospheric sciences with experience in the manipulation of large datasets and the transfer of data over networks and the Internet. Familiarity with the UNIX environment and the ability to work well as a member of a team are necessary. Oral and written communication skills are critical, as is familiarity with HTML web page development. Experience in operational environments is desirable.

Please send a one to two page statement of interest and availability, vitae, and names, addresses, and telephone numbers of three references to: Meg Austin, UCAR Visiting Scientist Programs, P.O. Box 3000, Boulder, CO 80307. For further information please call 303-497-8649 or send e-mail to vsp@ucar.edu.

\section{Application deadline is 1 September 1997}

UCAR is an Equal Opportunity/Affirmative Action employer UCAR strongly encourages applications from women and minorities 